\title{
Fault-tolerant Control based on Sliding Mode for Overactuated Electric Vehicles
}

\author{
António Lopes \\ INESC TEC \\ Faculty of Engineering, University of Porto \\ Porto, Portugal \\ antonio.lopes@fe.up.pt
}

\author{
Rui Esteves Araújo \\ INESC TEC \\ Faculty of Engineering, University of Porto \\ Porto, Portugal \\ raraujo@fe.up.pt
}

\begin{abstract}
This paper proposes a fault tolerant control (FTC) scheme based on sliding mode control for multi-motor electric vehicles. A design method of a sliding mode tracking controller with control allocation is developed based on the information provide by fault detection and identification (FDI) mechanism. The vehicles states yaw rate and longitudinal velocity are simultaneously controlled to track their references. A particular attention is given to study the effect of non-perfect fault estimation. The control allocation explore the over actuated system in order to redistribute the control effort when a fault occurs. Simulations in various driving scenarios with different faults are carried out with a high-fidelity, CarSim, full-vehicle model. Simulation results show the effectiveness of the proposed FTC approach.
\end{abstract}

Keywords-Fault Tolerant Control, Sliding mode control, Control Allocation, electric vehicles, stability control;

\section{INTRODUCTION}

The technological breakthrough in electric vehicles and the implementation of multi-motor architecture in ground vehicles created an opportunity to explore the advantages of control in over actuated systems [1],[2]. The fast transient response of electric motors as well as the interesting torque/speed characteristic, gives an exciting lead over the combustion motors. The capability to control precisely the accelerating/braking torque of each wheel independently is also an useful feature of multi motor vehicles which enhances the typical control strategies [3],[4].

Nevertheless the increasing the number of actuators leads to an increase of system faults [1]. Therefore, the need for a fault tolerant control that can recover the system in the presence of motor failure and ensures the stability of the vehicle is required. From the control point of view the over actuated systems provides an inherit plurality of solution which can be used to accommodate system faults and even complete actuator failure [5].

The loss of effectiveness in an in-wheel motor can lead to vehicle instability or deteriorate vehicle handling. For these reasons fault tolerant control (FTC) has been an emergent area in ground overactuated vehicles [3],[4],[5],[6] and[7]. In this paper, we propose a further improvement to [3] by introducing the effect of non-perfect fault reconstruction in the design of the sliding controller. The focus in this paper is to arrive to a suitable control for four wheel independent drive vehicles that can sustain multiple actuators fault by distributing the effort to the healthy actuators. To address this problem, it is exposed a control structure in fig. 1 that define the methodology based in a non-linear sliding mode control to design a robust controller able to guarantee system stability and robustness to system uncertainties and disturbances [3] [6].

The paper is organized as follows. In section II a brief introduction to the complete vehicle model with the information regarding the actuators fault is given. Section III explores the proposed control strategy, as well as the control law for the sliding mode controller and the control allocation method. The resulting effect from a deficient fault reconstitution is also studied in this section. The results obtained with the proposed control are presented in section IV. The final conclusions on the analysis and results are made in section $\mathrm{V}$.

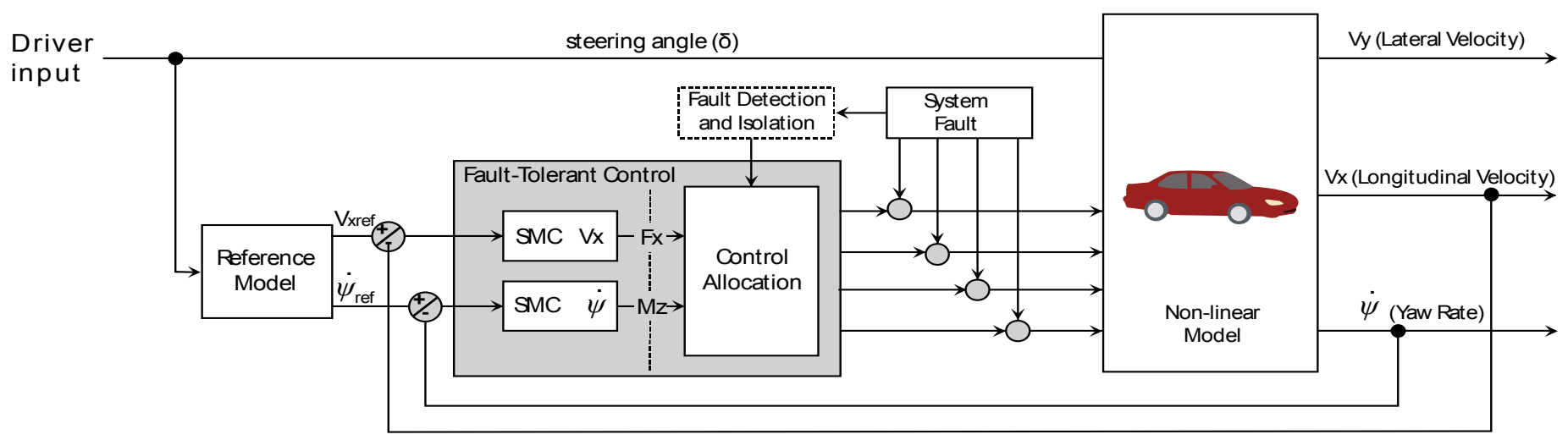

Fig. 1. Pronosed control structure for multi-motor electric vehicle 


\section{FAulty VehicLe Modeling}

\section{A. Vehical Planar model}

A model-based fault tolerant controller design requires a mathematical description of the vehicle. In this paper we are going to explore the planar model, which means that only the lateral and longitudinal dynamics are considered.

The study of the vehicle dynamics is defined by two key motions: the translation motion, and the rotational motion of the vehicle. Considering only the planar motion of the system it is possible to define the forces acting in the vehicle by the second law of Newton and the rotational moment by the Euler equation, obtaining the Newton-Euler equations of motion for a rigid vehicle[8]:

$$
\left\{\begin{array}{c}
\dot{v}_{X}=\frac{1}{m}\left(F_{X F L}+F_{X F R}+F_{X R L}+F_{X R R}-4 F_{R}-F_{\text {wind }}\right)+v_{Y} \cdot \dot{\psi} \\
\dot{v}_{Y}=\frac{1}{m}\left(2 C_{f}\left(\delta-\frac{v_{Y}}{v_{X}}-\frac{l_{f} \dot{\psi}}{v_{X}}\right)+2 C_{r}\left(\frac{l_{r} \dot{\psi}}{v_{X}}-\frac{v_{Y}}{v_{X}}\right)\right)-v_{X} \cdot \dot{\psi} \\
\ddot{\psi}=\frac{1}{I z}\left(2 l_{f} C_{f}\left(\delta-\frac{v_{Y}}{v_{X}}-\frac{l_{f} \dot{\psi}}{v_{X}}\right)-2 l_{r} C_{r}\left(\frac{l_{r} \dot{\psi}}{v_{X}}-\frac{v_{Y}}{v_{X}}\right)\right)+M_{z}
\end{array}\right.
$$

where the longitudinal vehicle speed $v_{x}$, the lateral vehicle speed $v_{y}$ and vehicle yaw rate $\dot{\psi}$ being the state variables of the nonlinear model. The mass of the system is defined as $m$ and the inertial moment of the vertical axis is $I Z$. The $M_{Z}$ defines the external moment component generated from the longitudinal forces applied in which wheel can be written as [9]:

$$
M_{Z}=\left(F_{X F R}-F_{X F L}+F_{X R R}-F_{X R L}\right) \frac{l_{s}}{2}
$$

This system can be exploited in the matrix form as presented in (3).

$$
\begin{gathered}
{\left[\begin{array}{l}
\dot{v}_{Y} \\
\dot{v}_{X} \\
\ddot{\psi}
\end{array}\right]=f(x)+C . \delta+B . u} \\
f(x)=\left[\begin{array}{l}
f 1(x) \\
f 2(x) \\
f 3(x)
\end{array}\right]=\left[\begin{array}{c}
a_{1} v_{Y}+a_{2} \dot{\psi} \\
\frac{1}{m}\left(-4 F_{R}-F_{\text {wind }}\right) \\
a_{3} v_{Y}+a_{4} \dot{\psi}
\end{array}\right]
\end{gathered}
$$

where $u$ is the input variable of our system:

$$
u=\left[F_{X F L}, F_{X F R}, F_{X R L}, F_{X R R}\right]^{T}
$$

and $F_{R}$ and $F_{\text {Windx }}$ are respectively the rolling resistance force and the wind resistance force. A complete formulation of vehicle model is beyond the scope of this paper, but a detailed discussion can be found in [8],[9].

$$
\begin{gathered}
C=\left[\begin{array}{ccc}
c_{1} & 0 & c_{2}
\end{array}\right]^{T} \\
B=\left[\begin{array}{cccc}
0 & 0 & 0 & 0 \\
\frac{1}{m} & \frac{1}{m} & \frac{1}{m} & \frac{1}{m} \\
-\frac{l_{s}}{2 I z} & \frac{l_{s}}{2 I z} & -\frac{l_{s}}{2 I z} & \frac{l_{s}}{2 I z}
\end{array}\right]
\end{gathered}
$$

Whose $a_{i}$ and $\mathrm{c}_{i}$ parameters take the form:

$$
\begin{aligned}
a_{1}=-\frac{2\left(C_{f}+C_{r}\right)}{m v_{X}} & a_{2}=\frac{2\left(C_{r} l_{r}-C_{f} l_{f}\right)}{m v_{x}}-v_{x} \\
a_{3}=\frac{2\left(C_{r} l_{r}-C_{f} l_{f}\right)}{I z \cdot v_{X}} & a_{4}=-\frac{2\left(C_{r} l_{r}^{2}+C_{f} l_{f}^{2}\right)}{I z \cdot v_{x}} \\
c_{1}=\frac{2 C_{f}}{m} & c_{2}=\frac{2 C_{f} l_{f}}{I z}
\end{aligned}
$$

\section{B. Actuator Faults}

The previous mathematical model does not describe the consequence of actuator fault in the vehicle. Basically is a faultfree model. In order to modelling the effect of actuator faults, it will be assumed that the occurrence of a fault has a direct impact in the available torque of the actuator. In the literature three types of actuator faults are considered [5]. The first one is the additive fault where the actuator fault is modeled as additive unknown signals that are superposed onto the control signal. The second one is the loss-of-effectiveness of actuator and the third fault type is the actuator's control effect stuck-at-fixed-level. In this paper, we only considered the second type which is modeled by multiplying a factor to the control signal. Considering a matrix $K$ that represents the actuator fault as expressed in (5) [5].

$$
K=\operatorname{diag}\left(k_{F L}, k_{F R}, k_{R L}, k_{F R}\right)
$$

with, $k_{j w} \in[0,1]$, where $k_{j w}$ expresses loss-of-effectiveness of the $j w$ th actuator. The fault gain express the severity of the actuator fault ranging from 1 , which represent a complete failure of the actuator, to 0 that express the absence of fault [3]. Therefore, a mathematical model representing a faulty vehicle model can take the following form:

$$
\left[\begin{array}{l}
v_{Y} \\
v_{X} \\
\ddot{\psi}
\end{array}\right]=f(x)+C \cdot \delta+B(I-K) u .
$$

This state-space model is used for the controller design in the next section.

\section{FAUlt TOLERANT CONTROLLER DESIGN}

In this section, the design of FTC will be explains in details. The main objective of the design is to derive a control law for (6) and, at the same time, reduce relevant disturbances and mitigate the systems faults. However, available controller may not meet all requirements needed. In particular this work deepens the initial work with respect to the effect of non-perfect fault reconstruction.

The main purpose of the control is to track $V_{x r e f}$ and $\dot{\psi}_{\text {ref, }}$ treating the lateral speed as an unknown disturbance. In this paper the FDI system will not be discussed, however we will assume that the faults introduced in the system are promptly detected using a subsystem capable to detect and identify the origin of the fault. The information obtained by FDI is send to the proposed controller that is capable to reconfigure the control allocation in order to eliminate the actuator fault. The detection and isolation of the faults that occurs in the system is a relevant subject that will be studied in subsequent works. 


\section{A. Control Structure}

The input given by the driver is used to obtain the desirable yaw rate as well as the desirable speed of the vehicle. The input $u$ of the system (6) is assumed to be independent of the vehicle states. Thus, we substitute it by a virtual control variable to simplify the control design task and to decouple it from an upper-level controller to track the vehicle dynamics desired by the driver and a low level controller for individual wheel torque control allocation algorithm. The control of the state variables are treated as independent of each other enabling a decoupled study as well as a separated control of each variable. The control allocation is design to distribute the effort of the actuators in an optimal way and to eliminate the disturbance created by the actuators faults. Note that the wheel torque allocation algorithm is responsible to manage the system faults.

\section{B. Control Allocation}

The control allocation algorithm is a key element in the proposed fault tolerant control approach. The information obtained from the FDI subsystem will be considered perfect in this section.

Let $B \in \mathbb{R}^{n x m}$ and $m$ is the number of actuators of the system. The B matrix can be reordered as [5]:

$$
B=\left[\begin{array}{l}
B_{1} \\
B_{2}
\end{array}\right]
$$

With

$$
B_{2}=\left[\begin{array}{cccc}
\frac{1}{m} & \frac{1}{m} & \frac{1}{m} & \frac{1}{m} \\
-\frac{l_{s}}{2 I z} & \frac{l_{s}}{2 I z} & -\frac{l_{s}}{2 I z} & \frac{l_{s}}{2 I z}
\end{array}\right]
$$

where $B_{1} \in \mathbb{R}^{(\mathrm{n}-\mathrm{l}) \times \mathrm{m}}$ and $B_{2} \in \mathbb{R}^{1 \times \mathrm{m}}$ has rank 1 . The $B_{1}$ matrix is null which means that all the effort of the control is due to $B_{2}$ matrix.

The $B_{2}$ matrix can be transformed in such a way that $B_{2} B_{2}{ }^{T}=I_{l}$ and therefore $\left\|B_{2}\right\|=1$. This is always possible since $\operatorname{rank}\left(B_{2}\right)=l[5]$. The resulting matrix is defined in (9).

$$
B_{2}=\left[\begin{array}{cccc}
\frac{1}{2} & \frac{1}{2} & \frac{1}{2} & \frac{1}{2} \\
-\frac{1}{2} & \frac{1}{2} & -\frac{1}{2} & \frac{1}{2}
\end{array}\right]
$$

The control allocation and the control law are designed separately so it is necessary an intermediate virtual control. The transformation performed in (9) must be accommodated by defining a suitable virtual control variable:

$$
v=\left[\frac{m}{2} F_{X}, \frac{I_{Z}}{l s} M_{Z}\right]^{T}
$$

The virtual control variable $v$ can be expressed as a result of the control variable $u$ in (8) and it summarizes the total longitudinal force and the total yaw moment acting on the center of gravity of the vehicle.

$$
v=B_{2} u
$$

From direct manipulation of (11) gives [5]

$$
u=B_{2}^{\dagger} v
$$

where $B^{\dagger}$ represent the right pseudo-inverse matrix of $B_{2}$. The choice of the pseudo inverse matrix, $B^{\dagger}$ is obtained by solving a minimization problem [5]:

$$
\min _{u} u^{T} W^{-1} u \quad \text { Subject to } B_{2} u=v
$$

where $W \in \mathbb{R}^{\mathrm{mxm}}$ is a positive diagonal weighting matrix. The function in (13) minimizes the weighted sum of squares associated with the control vector $u$. The optimal solution is obtained with (14) [5].

$$
B_{2}^{\dagger}=W B_{2}^{T}\left(B_{2} W B_{2}^{T}\right)^{-1}
$$

\section{Weighting Matrix}

The weighting matrix $\mathrm{W}$ is chosen in order to:

1) Redistribute the control in the presence of faults in the system;

\section{2) Explore the tire load to improve the grip.}

The presence of faults can be explored by a fault matrix $K$ expressed in (5). So it is possible to define a weighting matrix $\mathrm{M}$ that exploit the actuator effectiveness [5].

$$
M=(I-K)
$$

The tire vertical load of each tire can be define as followed [9],[4]:

$$
\left\{\begin{array}{l}
F_{Z F L}=\frac{1}{2} \frac{l_{r}}{l} m \cdot g-\rho_{f} a_{Y} m \frac{h_{g}}{l_{s}}-a_{X} m \frac{h_{g}}{l} \\
F_{Z F R}=\frac{1}{2} \frac{l_{r}}{l} m \cdot g+\rho_{f} a_{Y} m \frac{h_{g}}{l_{s}}-a_{X} m \frac{h_{g}}{l} \\
F_{Z R L}=\frac{1}{2} \frac{l_{r}}{l} m \cdot g-\rho_{r} a_{Y} m \frac{h_{g}}{l_{s}}+a_{X} m \frac{h_{g}}{l} \\
F_{Z R R}=\frac{1}{2} \frac{l_{r}}{l} m \cdot g+\rho_{r} a_{Y} m \frac{h_{g}}{l_{s}}+a_{X} m \frac{h_{g}}{l}
\end{array}\right.
$$

where $\rho_{f}$ and $\rho_{r}$ are respectively the front and rear roll coefficient, $h_{g}$ is the height of the center of gravity of the vehicle.

There is a direct relationship between the longitudinal force $F_{X}$, the lateral force $F_{Y}$ and the vertical force $F_{Z}$ that must be satisfied [9],[4]:

$$
\sqrt{{F_{X}}^{2}+{F_{Y}}^{2}} \leq \mu F_{Z}
$$

where $\mu$ is the friction coefficient of the road. From (17) is noticeable that the magnitude of the force that the tire generates in contact with the road should not exceed the product of the vertical load of the tire and the friction coefficient. The vector created by the result of the two components of the tire/road must be restricted to the friction circle with $\mu F_{Z}$ radius [9], [4].

The workload of each tire can be expressed as shown in (18) by considering that the lateral forces that the tire originates are negligible $\left(F_{Y j w} \ll F_{X j w}\right)$. 


$$
\begin{array}{ll}
n_{F L}=\frac{F_{X F L}}{\mu \cdot F_{Z F L}} ; & n_{R R}=\frac{F_{X F R}}{\mu \cdot F_{Z F R}} ; \\
n_{R L}=\frac{F_{X R L}}{\mu \cdot F_{Z R L}} ; & n_{R R}=\frac{F_{X R R}}{\mu \cdot F_{Z R R}} ;
\end{array}
$$

A weighting matrix $\mathrm{J}$ regarding the tire workload can be expressed as [3]:

$$
J=\operatorname{diag}\left(\left(\mu . F_{Z F L}\right)^{2},\left(\mu . F_{Z F R}\right)^{2},\left(\mu \cdot F_{Z R L}\right)^{2},\left(\mu \cdot F_{Z R R}\right)^{2}\right)
$$

The final weighting matrix $\mathrm{W}$ can be obtained by combining the M matrix (15) relative to the system faults with the load restriction [3],[4] obtaining:

$$
\begin{gathered}
W=\operatorname{diag}\left(\left(1-k_{F L}\right)\left(\mu \cdot F_{Z F L}\right)^{2},\left(1-k_{F R}\right)\left(\mu \cdot F_{Z F R}\right)^{2},(1-\right. \\
\left.\left.k_{R L}\right)\left(\mu \cdot F_{Z R L}\right)^{2},\left(1-k_{R R}\right)\left(\mu \cdot F_{Z R R}\right)^{2}\right)
\end{gathered}
$$

\section{Non-Perfect Fault Reconstruction}

Until this point the fault reconstruction was assumed as perfect, now it will be exposed the effect of an imperfect reconstruction of the system fault.

Consider now $\bar{K}$ as the estimated actuator efficiency based on the FDI information and we suppose that $\bar{K} \neq K$. Thus it is possible to define the faults weighting matrix as [5]:

$$
\bar{M}=I-\bar{K}
$$

By assuming $\Delta=\operatorname{diag}\left\{\Delta_{F L}, \Delta_{F R}, \Delta_{R L}, \Delta_{R R}\right\}$, which represents the level of imperfection of the fault reconstruction, the actual faults weighting matrix can be expressed as [5]:

$$
M=(I-\Delta) \bar{M}
$$

The weighting matrix $M$ was defined in (19). By replacing the result (22) in the model (6) results the effect of the nonperfect fault reconstruction [5]:

$$
\left[\begin{array}{c}
\dot{v}_{Y} \\
\dot{v}_{X} \\
\ddot{\psi}
\end{array}\right]=f(x)+C \cdot \delta+B(I-\Delta) \bar{M} \cdot B_{2}{ }^{\dagger} v
$$

By defining $\bar{v}=\left(B_{2} \bar{W} B_{2}{ }^{T}\right)^{-1} v$ the error in fault reconstruction becomes explicit [5]:

$$
\begin{aligned}
{\left[\begin{array}{c}
\dot{v}_{Y} \\
\dot{v}_{X} \\
\ddot{\psi}
\end{array}\right]=f(x)+C . \delta } & +\left[\begin{array}{c}
B_{1} B_{2}{ }^{T} \\
I
\end{array}\right] \bar{v}-\left[\begin{array}{l}
B_{1}\left(I-\bar{M}^{2}\right) B_{2}{ }^{T} \\
B_{2}\left(I-\bar{M}^{2}\right) B_{2}{ }^{T}
\end{array}\right] \bar{v} \\
& -\left[\begin{array}{c}
B_{1} \Delta \bar{M}^{2} B_{2}{ }^{T} \\
B_{2} \Delta \bar{M}^{2} B_{2}{ }^{T}
\end{array}\right] \bar{v}
\end{aligned}
$$

In the system expressed in (24) is evident the presence of an uncertainty that can be explicitly defined as:

$$
\left[\begin{array}{c}
\Delta v_{Y} \\
\Delta v_{X} \\
\Delta \dot{\psi}
\end{array}\right]=\left[\begin{array}{l}
B_{1} \Delta \bar{M}^{2} B_{2}{ }^{T} \\
B_{2} \Delta \bar{M}^{2} B_{2}{ }^{T}
\end{array}\right] \bar{v}
$$

\section{E. Sliding mode Control-yaw rate}

From the algebraic manipulation of the model presented in (6) results (26) [7].

$$
\begin{gathered}
I z . \ddot{\psi}=-\frac{2\left(l f^{2} C f+l r^{2} c r\right)}{v_{X}} \dot{\psi}+2 . l f . C f \delta-2(l f . C f- \\
l r . C r) \frac{v_{Y}}{v_{X}}+M_{\text {Zext }}+\Delta \dot{\psi}
\end{gathered}
$$

Where $\Delta \dot{\psi}$ represents the effect from a non-perfect reconstruction defined in (25).

The sliding variable will be defined as (27) in the proposed yaw rate control [7].

$$
\sigma=\dot{\psi}_{r e f}-\dot{\psi}
$$

The total bounded disturbances in the system can be defined as:

$$
\vartheta(x, t)=\ddot{\psi}_{r e f}-\frac{2(l f . C f-l r . C r) v_{Y}}{I z \cdot v_{X}}-\frac{\Delta \dot{\psi}}{I z}
$$

The control law chosen is defined as a result of two complementary control laws [10]:

$$
M_{\text {Zext }}=\left(u_{e q}+u_{d}\right)
$$

One component is the equivalent control law which is the control function necessary after reaching the sliding surface $\sigma=0$ to ensure that the system remains in the trajectory [10].

$$
u_{e q}=-\frac{2\left(l f^{2} c f+l r^{2} c r\right)}{v_{X}} \dot{\psi}+2 . l f . C f . \delta
$$

The second component is chosen to eliminate the bonded disturbances defined in (28) and can be selected as (31) [10],[7].

$$
u_{d}=[K p . s+K s \cdot \operatorname{sign}(s)] . I z
$$

To prove the stability of the proposed control we used the following Lyapunov equation [10]:

$$
V=\frac{1}{2} \sigma^{2}
$$

Once the control law is completely defined it is possible to prove the stability of the system by substituting the control law in the equation (29) in the derivation of Lyapunov function defined in (32) resulting the condition (33).

$$
\dot{V}=|\sigma|(T-K s)-K p . \sigma^{2}<0
$$

From the condition in (33) it is clear that the stability is reach by choosing $K s>0$ which is the control parameter, that is tuned to eliminate the bounded disturbance $\mathrm{T}$, and $K p>0$, this last parameter define the converge rate of the controller [7]. 
TABLE I. PARAMETERS OF THE VEHICLE MODEL FROM CARSIM

\begin{tabular}{cc}
\hline \hline Vehicle Parameter & Value \\
\hline Cf-Cornering stiffness front & $24.5 \mathrm{kN} / \mathrm{rad}$ \\
Cr-Cornering stiffness rear & $23.1 \mathrm{kN} / \mathrm{rad}$ \\
Iz-Vehicle inertia around z-axis & $1130 \mathrm{~kg} \mathrm{~m} \mathrm{~m}^{2}$ \\
$m-$ Vehicle mass & $830 \mathrm{~kg}$ \\
lf-Distance from COG to front axle & $1.103 \mathrm{~m}$ \\
lr - Distance from COG to rear axle & $1.244 \mathrm{~m}$ \\
ls - Width of the vehicle & $1.78 \mathrm{~m}$ \\
\hline
\end{tabular}

\section{RESULTS AND DISCUSSION}

The numerical experimental has done based on the car of class A model. Table I shown the parameters used for the numerical experimental proposed. The controller was developed in Matalab-Simulink environment. The real parameters are imported from CarSim to Simulink then exported to the CarSim as a feedback signal from the controller. To evaluate the performance of the proposed control three different simulations were performed: a J-turn, a Double Line change and a Single Line change. In the referred simulation are presented extreme situations of road friction and actuators faults in order to infer about the controller performance.

\section{A. J-turn}

In this maneuver there is a first segment in straight line followed by a J-turn of 45 degrees. To evaluate the control response to different friction coefficients this maneuver is carried with a split $\mu$, having a lower friction coefficient in the left side of the road $(\mu=0.1)$ and a higher coefficient in the right side $(\mu=0.5)$.

During the straight line it is introduce a fault in the front left tire and the effectiveness of that motor decreases $10 \%$ per second from the first second until the end of the maneuver finishing with only $30 \%$ of effectiveness. The yaw rate follows the reference with the proposed control as opposed to the system without control as seen in Fig. 2.

The dynamic of the longitudinal velocity is also explored, by raising the vehicle speed during the straight line and maintaining the speed in the turn (see Fig. 3). The initial speed is $45 \mathrm{Km} / \mathrm{h}$ entering the curve at $50 \mathrm{Km} / \mathrm{h}$.

The effort of each motor in the maneuver is presented in Fig. 5. At $t=0.5 \mathrm{~s}$, the applied torque rises to satisfy the increase of longitudinal speed. The fault applied in the first second decreases the effectiveness of the motor in the rear right wheel, as a result the motor of the front right wheel increases the torque. The J-turn initiated at $t=5 \mathrm{~s}$ removes the controller from the sliding surface, but it rapidly returns to the surface at $t=5.5 \mathrm{~s}$ as it is shown.

\section{B. Double line change}

In this second simulation it is explored a more difficult maneuver with low friction coefficient $(\mu=0.3)$. The system is exposed to two faults in two different actuators in order to expose the performance of the control allocation system. The

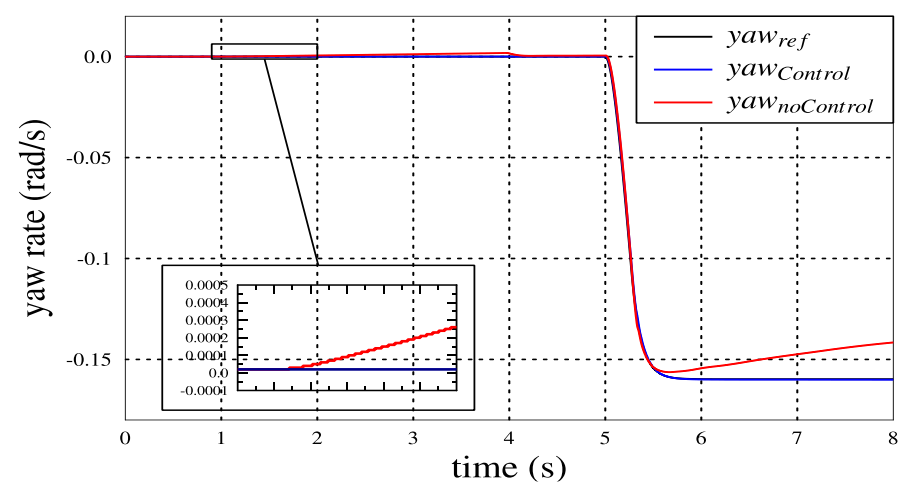

Fig. 2. Yaw rate in J-turn manuever

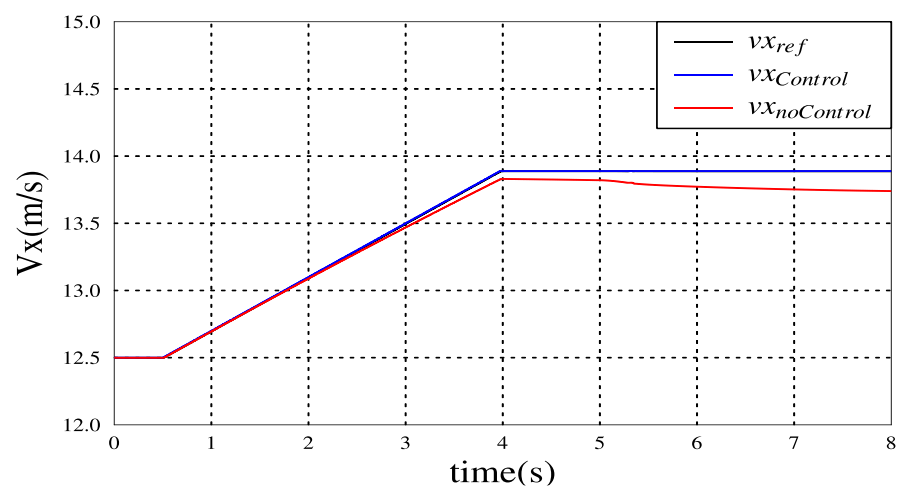

Fig. 3. Vehicle longitudinal speed in J-turn manuever

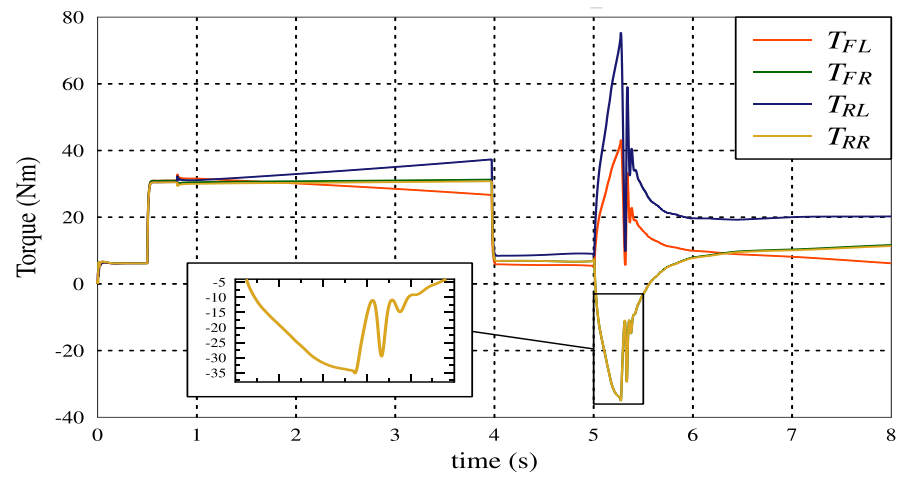

Fig. 4. Torque allocation in J-turn manuever

vehicle speed is maintain unchanged during the entire maneuver with a constant speed of $60 \mathrm{Km} / \mathrm{h}$.

At $t=2.5$ it is introduced a fault in the actuator of the front left wheel. Fig 5 shows the yaw rate for the FTC control and without control. It is obvious in Fig. 5 that the vehicle yaw rate can precisely track the desired reference expected by the driver with control. Without control the system cannot assure the desired yaw rate especially if the road friction coefficient decreases. The effect of the fault in the vehicle global position is obvious and shows the importance of the proposed control (see in Fig. 6).

The presence of the fault at $t=2.5 \mathrm{~s}$ is equalized by the increase of the torque applied in rear tire actuator. Fig. 7 shows the motor torque in the front left wheel decreases his 


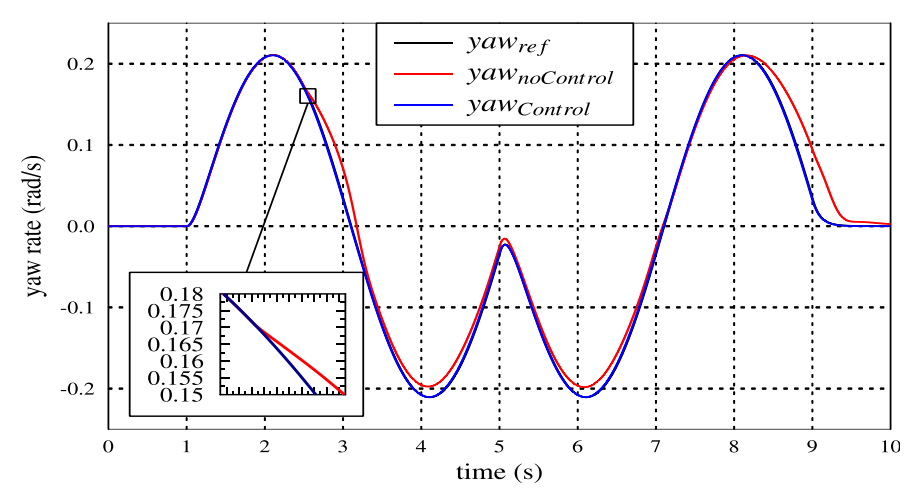

Fig. 5. Yaw rate in DLC manuever

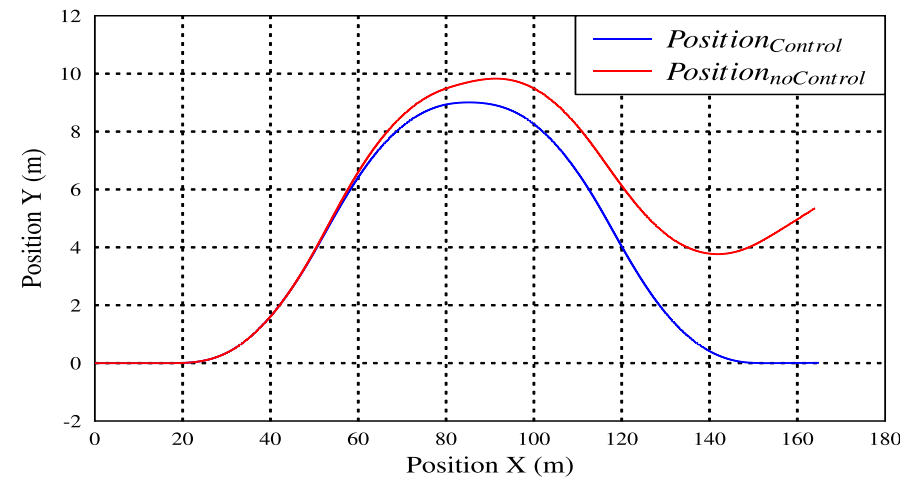

Fig. 6. Vehicle Global position in DLC manuever

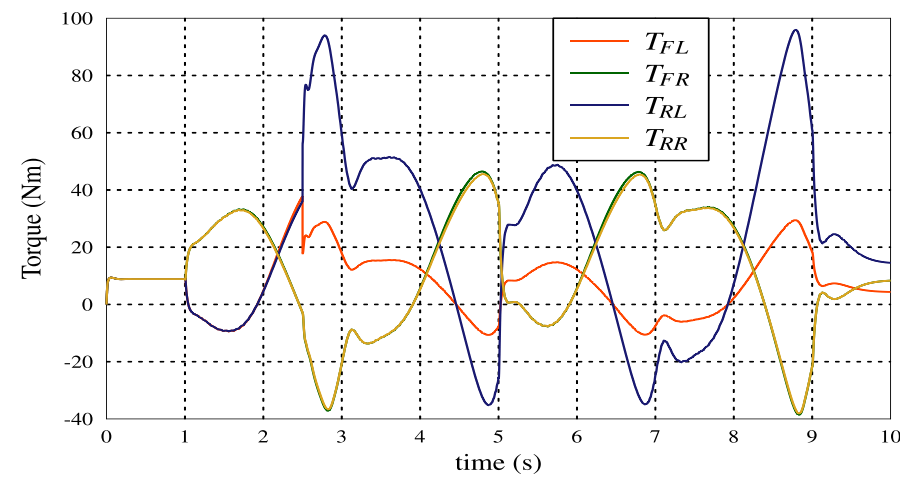

Fig. 7. Torque allocation in DLC manuever

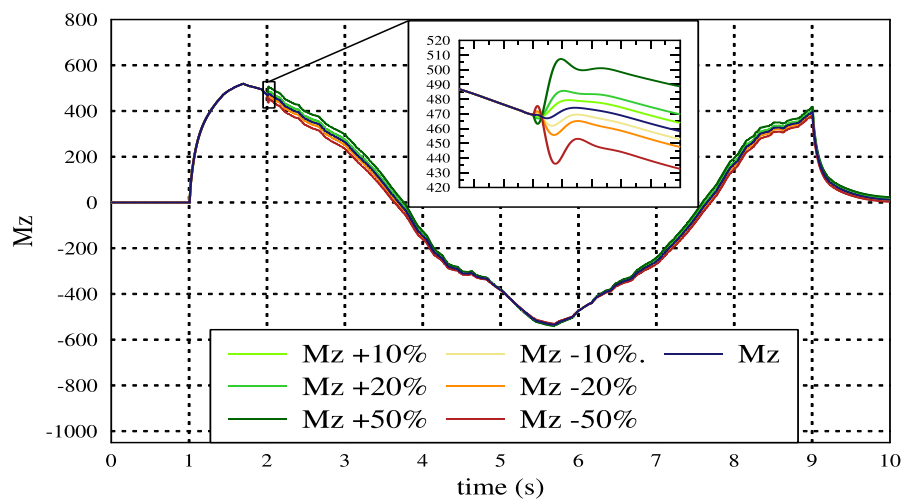

Fig. 8. Mz variation to fault imperfetion in SLC manuever

effectifeness to $30 \%$ and the motor in rear left wheel respond to the fault by increasing its torque.

\section{Single line change}

In this maneuver the vehicle changes line at $90 \mathrm{Km} / \mathrm{h}$ in a dry road $(\mathrm{u}=0.85)$ and it is explored the effect of the non-perfect fault reconstruction of the FDI subsystem by imposing a level of imperfection of $\pm 10 \%, \pm 20 \%$, and $\pm 50 \%$. The resulting virtual control imposed by the controller is presented in Fig. 8. The fault occours at $t=2 \mathrm{~s}$ in the motor of the front right wheel, at this time the actuator lost $80 \%$ of effectivness. The yaw rate and the speed of the vehicle follows the reference perfectly even in the presence of a $50 \%$ imperfection. Fig. 8 demonstrates the robustness of the controller to disturbances. The desired yaw rate does not change in the presence of these uncertainties as long as the actuators do not reach their limitations.

\section{CONCLUSION AND FUTURE WORK}

The present paper explore and validate the Fault Tolerant Control structure proposed based in sliding mode control for four-wheel independent drive vehicles. The faulty model was completely defined as well as the effect of a non-perfect reconstruction of the faults. The control redundancy is explored in such a way that the actuator fault do not influence the system overall performance, by redistributing the control effort to the remaining actuators and by exploring the tire with the most vertical load, improving the overall performance of the system. Numerical simulations have shown the performance of the proposed controller. Additionally, an investigation for the application fault detection and identification will be in focus of our future work.

\section{REFERENCES}

[1] J. Wang, and R. G. Longoria. "Coordinated and Reconfigurable Vehicle Dynamics Control," IEEE Transactions on Control Systems Technology, Vol. 17, No. 3, pp. 723 - 732, 2009.

[2] A.Silveira, R. E. Araújo, and R. Castro, "Survey on faulttolerantdiagnosis and control systems applied to multi-motor electric vehicles," in Technological Innovation for Sustainability (S. B. Heidelberg, ed.),pp. 359-366, 2011.

[3] A. Sérgio, and R. E. Araujo. "Fault-tolerant control using sliding mode techniques applied to multi-motor electric vehicle." Industrial Electronics Society, IECON 2013-39th Annual Conference of the IEEE. IEEE, 2013.

[4] N. Ando and H. Fujimoto, "Yaw-rate control for electric vehicle with active front/rear steering and driving/braking force distribution of rear wheels," in Advanced Motion Control, 2010 11th IEEE International Workshop on, pp. 726-731, IEEE, 2010

[5] H. Alwi, C. Edwards, and C. P. Tan, Fault detection and fault-tolerant control using sliding modes. Springer, 2011.

[6] R. Wang, and J. Wang. "Fault-tolerant control for electric ground vehicles with independently-actuated in-wheel motors." Journal of Dynamic Systems, Measurement, and Control 134.2 (2012): 021014.

[7] K. Nam, et al. "Design of adaptive sliding mode controller for robust yaw stabilization of in-wheel-motor-driven electric vehicles." EVS26, Electric Vehicle Symposium. 2012.

[8] R. N. Jazar, Vehicle dynamics: theory and application. Springer, 2008.

[9] U. Kienecke, and L. Nielsen, Automotive control systems. Warrendale, PA: Society of Automotive Engineers, 2000. 432 (2000).

[10] C. Edwards, L. Fridman, and A. Levant. Sliding mode control and observation. Birkhäuser, 2014. 